\title{
TAGUNG
}

\section{Demokratieförderung durch die Europäische Union zwischen Anspruch und Wirklichkeit}

\author{
Sonja Grimm*
}

Nicht nur gegenüber den Staaten Mittel- und Osteuropas betrieb beziehungsweise betreibt die Europäische Union (EU) dezidiert die Förderung von Rechtsstaatlichkeit, Demokratie und ,gutem Regieren'. Die Mittelmeeranrainer, Handelspartner in Lateinamerika und in Asien sowie verschiedene Staaten in Afrika gehören zu den Adressaten direkter und indirekter europäischer Demokratieförderung. Instrumente, Strategien und Wirkung dieses Demokratieexports sind jedoch bisher kaum untersucht. Die Konferenz ,The EU as an External Democracy Promoter" brachte regionale Spezialisten und Integrationsforscher zusammen, um folgende Fragen zu beantworten: Inwieweit nutzt die Europäische Union unterschiedliche Strategien der Demokratieförderung in verschiedenen Ländern und Kontexten? Welches sind die Kriterien für die Wahl bestimmter Förderinstrumente? Wie erfolgreich sind die gewählten Strategien und Instrumente für die externe Unterstützung von Demokratisierung, Demokratiestabilität und ,gutem Regieren"?

Die beiden Organisatorinnen, Michèle Knodt und Annette Jünemann, schlugen einleitend drei zentrale Variablen zur Erklärung der Strategiewahl der Europäischen Union in der Demokratieförderung vor: erstens das Handlungspotenzial der EU, bei dem die macht-, sicherheits- und wirtschaftspolitischen Interessen der einzelnen Mitglieder gegenüber dem Zielland eine große Rolle spielen; zweitens die Ressourcenrelation zwischen der EU und dem Drittstaat; und drittens die Resonanzstruktur des Drittstaats, sprich die Bereitschaft der relevanten Eliten, die Demokratieförderung anzunehmen. Die beiden zu
The EU as an External Democracy Promoter: East and Central Europe, the Mediterranean, Asia, Latin America, the Caribbean, Africa and Pacific in Comparison

Internationale Konferenz des Arbeitskreises Europäische Integration (AEI) und der Technischen Universität Darmstadt mit Unterstuitzung der Deutschen Forschungsgemeinschaft (DFG) und des CONNEXExzellenznetzwerks

Darmstadt, 18./19. November 2005

\section{Wissenschaftliche Leitung}

Prof. Dr. Michèle KNODT, Technische Universität (TU) Darmstadt

Prof. Dr. Annette JÜNEMANN, HelmutSchmid-Universität der Bundeswehr Hamburg

Welcome and introduction: Conceptualising the EU's Promotion of Democracy Prof. Dr. Michèle KNODT, Prof. Dr. Annette JÜNEMANN

\section{Africa}

Challenging cases: EU democracy promotion in South Africa and the DR Congo

Prof. Dr. Siegmar SCHMIDT, Universität Landau

Democracy Promotion of the EU and France in Westafrica: Ivory Coast and Burkina Faso

Prof. Dr. Stefan BRÜNE, Deutsches Übersee-Institut (DUI), Hamburg

Evaluating EU Democracy Promotion in Ghana

Dr. Gordon CRAWFORD, University of Leeds

\footnotetext{
* Sonja Grimm, Wissenschaftliche Mitarbeiterin, Abteilung „Demokratie: Strukturen, Leistungsprofil und Herausforderungen“, Wissenschaftszentrum Berlin für Sozialforschung.
} 
prüfenden Thesen lauteten: Die jeweilige Ausprägung der genannten Variablen markiert spezifisch die Handlungsfähigkeit der Union und beeinflusst die Auswahl der Förderstrategie. Bisher nicht berücksichtigte sicherheitspolitische Erwägungen können sich jedoch im Einzelfall als zentrale Störvariablen erweisen.

Mäßiger Erfolg in Afrika mangels Interesse und geeigneter Instrumente

Siegmar Schmidt präsentierte im ersten Vortrag der Tagung die Demokratieförderung der Europäischen Union in Südafrika und der Demokratischen Republik Kongo. Während des Apartheidregimes war die Politik der Europäischen Union gegenüber Südafrika vor allem durch Zurückhaltung geprägt. Allein das Sonderprogramm für die Opfer des Apartheidregimes von 1986 kann laut Schmidt als nennenswerter Beitrag zur Sanktionierung des Regimes bezeichnet werden. Nach Ende des Apartheidregimes habe sich die Europäische Union vor allem als Beobachter der Wahlen von 1994 hervorgetan. Im Rahmen des Programms für Wiederaufbau und Entwicklung zahlt die Europäische Union von 2000 bis 2006 rund 880 Millionen Euro vor allem für Infrastrukturmaßnahmen und zivilgesellschaftliche Projekte. Allerdings ist Schmidt skeptisch hinsichtlich der Wirksamkeit dieses Programms. Die Verzögerung der Implementierung des Freihandelsabkommens von 1999 um fünf Jahre habe Südafrika mehr geschadet als die bis 2006 gewährten Gelder nutzen könnten. Die Verhältnisse in der Demokratischen Republik Kongo wiederum seien ein Beispiel für die Hilflosigkeit der internationalen Gemeinschaft, eine geeignete Strategie zur Lösung des dortigen Konflikts zu entwickeln und die überzogenen Erwartungen, Demokratie in ein bürgerkriegsgeschütteltes Land exportieren zu können.

Stefan Brüne machte in seinem Vortrag auf die desaströsen Zustände in Äthiopien aufmerksam, die in der Öffentlichkeit nicht genügend wahrgenommen würden. Gerade die Europäische Union, die sonst ihr besonderes
II. East and Central Europe (incl. former Soviet Union)

EU Democracy Promotion in Belarus, Ukraine, Moldova

Dr. Iris KEMPE, Centrum für angewandte Politikforschung (CAP), München

The Role of Civil Society in Promoting Democracy: West-Balkan and the Baltics in Comparison

Dr. Susan STEWART, Mannheimer Zentrum für Europäische Sozialforschung, Universität Mannheim

The Role of the Promotion of Democracy in the New Neighbourhood Policy

Dr. Arkady MOSHES, Finish Institute of International Affairs (FIIA), Helsinki

EU-Russia: A Special Relationship?

Dr. Katrin BASTIAN, Humboldt-Universität zu Berlin

\section{Latin America and the Caribbean}

Europe-Latin American City Networks. Building a Strategy from Below

Rainer ROTHFUSS, Universität Tübingen

EU-Latin America relationship: The Examples of Columbia, Cuba, Venezuela

Dr. Susanne GRATIUS, Stiftung Wissenschaft und Politik, Berlin; Fundación para las Relaciones Internacionales y el Diálogo Exterior (FRIDE), Madrid

IV. Asia

EU Democratisation Policy in Afghanistan

Dr. Florian P. KÜHN, Helmut-Schmidt-Universität der Bundeswehr Hamburg

Democracy Promotion of the EU in Myanmar/ Burma and China

Dr. Franco ALGIERI, CAP, München

V. The Mediterranean and Arab world

Explaining the Incoherence of EU-Democracy Promotion in the Mediterranean. A Constructivist Approach that draws on Role Theory

Prof. Dr. Annette JÜNEMANN

The European Union as a Promoter of Democracy in a Conflict Ridden Sub-Region: The Case of the Middle East

Dr. Richard YOUNGS, FRIDE, Madrid

EU Promotion of Human Rights in the Mediterranean - a Practitioner's View

Dr. Marc SCHADE-POULSEN, Euro-Mediterranean Human Rights Network, Copenhagen

Pre-Accession and Neighbourhood: European Union Democratic Conditionality

Elena BARACANI, Università degli studi di Firenze

Summing up of the Conference: First Comparative Results and Revised Hypotheses

Prof. Dr. Annette JÜNEMANN, Prof. Dr. Michèle KNODT 
Augenmerk auf Werte wie Demokratie, Frieden und Menschenrechte richte, versage hier völlig. Ihre wirtschaftlichen Sanktionen zeigten keinerlei Wirkung auf die Konfliktparteien und seien als Konfliktlösungsinstrument ungeeignet. Diese Aussage veranlasste die Teilnehmer der Tagung zu einer Diskussion darüber, ob eine negative Konditionierung in Form ökonomischer Sanktionen überhaupt geeignet ist, Demokratie zu fördern.

Gordon Crawford widmete sich in seiner Analyse der Demokratieförderung in Ghana. $\mathrm{Zu}$ seiner Verwunderung ist diese praktisch nicht existent. Gerade die stabile politische Situation und die signifikanten Fortschritte in der Konsolidierung demokratischer Strukturen einerseits sowie der hohe Bedarf an wirtschaftlicher Entwicklung andererseits böten ein günstiges Umfeld dafür, den Erfolg von Demokratieförderung in Afrika einmal grundsätzlich testen zu können. Würde sich Demokratieförderung in Ghana trotz des günstigen Umfelds als nicht erfolgreich erweisen, dann könne sie im gesamten afrikanischen Kontext nicht funktionieren, lautete Crawfords Argument.

Fehlende Beitrittsperspektive blockiert Entwicklung der osteuropäischen Nachbarn

Über die Bedeutung der europäischen Nachbarschaftspolitik gegenüber der Ukraine, Moldau und Weißrussland sprach Iris Kempe. Die neue Nachbarschaftspolitik solle in den Augen der EU den Rahmen für eine Kooperation mit den im Osten an die Union angrenzenden Staaten schaffen, ohne zu großzügig eine Unionsmitgliedschaft zu offerieren. Die nicht vorhandene Mitgliedschaftsperspektive erweist sich laut Kempe als ein schwer wiegendes Hindernis auf dem ukrainischen und moldauischen Weg zur Demokratie. Eine Kooperation mit Weißrussland stehe für die Union zu Recht so lange nicht auf der Tagesordnung wie das diktatorische Regime Lukaschenkos an der Macht bleibe.
Ist die Mitgliedschaftsperspektive tatsächlich die einzige erfolgreiche Demokratisierungsstrategie, die die Europäischen Union anzubieten hat? Folgt man den Thesen von Arkady Moshes, muss man zu diesem Schluss kommen. In seiner umfassenden Bewertung der europäischen Nachbarschaftspolitik kritisierte er vor allem die Beschränkung auf die wirtschaftliche Zusammenarbeit zwischen der Europäischen Union und den osteuropäischen Nichtmitgliedern, die nicht genügend Anreize für eine national gesteuerte Demokratisierung bereitstelle. Auch die Aktionspläne der Europäischen Union trügen nicht zu einer effektiven Implementierung demokratischer Strukturen bei. Die Union konzentriere sich auf Stabilität, Sicherheit und Prosperität und setze ihre Maßnahmen zu bürokratisch und zu langsam um, um den Übergang zur Demokratie sinnvoll unterstützen zu können. Geoffrey Harris entgegnete in der Diskussion, dass man von der europäischen Nachbarschaftspolitik kaum Leistungen erwarten könne, die die Union selbst für die erfolgreich konsolidierten mittelosteuropäischen Staaten nicht erbracht habe. In keinem Falle könne die Union einen politischen Regimewechsel aktiv betreiben.

Katrin Bastian erläuterte anschließend die Haltung der Europäischen Union gegenüber Russland. Das Partnerschafts- und Kooperationsabkommen, die Bastians Ansicht nach überschätzte Gemeinsame Strategie gegenüber Russland und die Einbindung Russlands in die technische Hilfe bei Verwaltungs- und Wirtschaftsreformen über das TACIS-Programm könnten nicht darüber hinwegtäuschen, dass die Union gegenüber Russland nur sehr vorsichtig auf die Umsetzung demokratischer Reformen dränge. Bastian beobachtet eine besondere Beziehung zwischen der Europäischen Union und Russland, die sich von den Verbindungen zu und Erwartungen an die anderen osteuropäischen Partner deutlich unterscheide. Bastian erklärte dies mit der besonderen historischen Verantwortung der EU gegenüber Russland, der geografischen Nähe, der institutionalisierten Dialogstruktur und dem differierenden Demokratieverständnis. 
Susan Stewart referierte erste Ergebnisse aus ihrem Forschungsprojekt „The Role of Civil Society in Promoting Democracy: West-Balkan and the Baltics in Comparison". Stewart evaluiert darin mit einem eigenen Kriterienkatalog, wie erfolgreich Demokratieförderung ist, wenn sie mittels einer gezielten Unterstützung zivilgesellschaftlicher Akteure erfolgt. Bei der Bewertung der europäischen Programme stellt Stewart starke konzeptionelle Kontinuitäten zwischen dem PHARE-Programm und den beiden jüngeren Programmen CARDS und EIDHR fest. Bei der Finanzierung und dem Ablauf der Mittelvergabe bedürften auch die neueren Programme weiterer Verbesserung. Verstärkt hätten sich hingegen die Flexibilität der Programme sowie die Betonung des Nachhaltigkeitsaspekts.

Partnerschaft und Frieden, nicht Demokratie stehen gegenüber Lateinamerika im Mittelpunkt

Rainer Rothfuss brachte den Teilnehmern der Konferenz in seinem Vortrag Vorzüge und Nachteile europäisch-lateinamerikanischer Städtenetzwerke näher. Die dezentrale Kooperationsstruktur des URB-Al-Programms, so seine These, biete den beteiligten Städten und Kommunen Gelegenheit, gegenseitig aus erfolgreichen Stadtentwicklungsprojekten $\mathrm{zu}$ lernen und in 13 verschiedenen Themenbereichen lokale Partizipation und Eigenverantwortung einzuüben. Die Europäische Union sei aufgrund ihrer ausgefeilten institutionellen Struktur, ihrer langjährigen Erfahrung mit Städtepartnerschaften und ihrem Interesse an einer strategischen Kooperation mit Lateinamerika eine besonders geeignete Initiatorin solcher Städtenetzwerke.

Im Gegensatz zu Rothfuss schätzt Susanne Gratius die Rolle der Europäischen Union in Lateinamerika und vor allem in Kolumbien, Kuba und Venezuela als nicht besonders bedeutend ein. Die fehlende gemeinsame Strategie der Europäischen Union gegenüber den genannten Ländern und die Fokussierung auf Friedensbildung und die Verbreitung der
Menschenrechte marginalisiere die Forderung nach Demokratie stützenden Reformen, obwohl diese in allen Kooperationsverträgen als Demokratieklausel enthalten sei. Einen Anstoß zu Liberalisierung und Demokratisierung durch diese Klausel konnte Gratius nicht feststellen. Verglichen mit dem Einflusspotenzial der USA in der Region, werde die Europäische Union auch in Zukunft in Lateinamerika ein unbedeutender Akteur bleiben.

\section{Heterogene Kontexte erfordern differenzier- tere Strategien im asiatischen Raum}

Nicht zuletzt der Asienabschnitt der Konferenz machte die Notwendigkeit deutlich, bei der Untersuchung der europäischen Förderstrategien stärker nach den Niveaus der Demokratisierungsfortschritte in einzelnen Ländern zu differenzieren und sich dabei über regionale Kontexte hinwegzusetzen.

Florian P. Kühn erläuterte, warum die Europäische Union in Nachkriegsgesellschaften die Mammutaufgabe des Wiederaufbaus und der Friedenskonsolidierung nach einem (Bürger-)Krieg keinesfalls allein bewältigen könne. Das Beispiel Afghanistan zeige, dass die Europäische Union gemeinsam mit den Vereinten Nationen vor allem im Aufbau der Gesundheitsversorgung sowie bei der Gewährung ökonomischer Unterstützung einen wichtigen Beitrag leiste. Allerdings bedürfe es in Afghanistan auch des Aufbaus demokratischer Institutionen, was - wie der in seiner Form einzigartige Bonn-Prozess gezeigt habe - eine langfristige und komplexe Aufgabe sei. Eine spezifische Demokratisierungsstrategie verfolgt die Europäische Union Kühns Einschätzung nach in Afghanistan zurzeit nicht.

Auf eine gänzlich andere Problemlage ging Franco Algieri in seinem Vortrag über die Haltung der Europäischen Union gegenüber Myanmar/Burma und China ein. Er machte darauf aufmerksam, dass sich hinter jeder noch so gut gemeinten Menschenrechts- und Demokratieförderpolitik harte wirtschaftliche Interessen verbürgen. Gerade in der europäi- 
schen Chinapolitik werde das deutlich. Zwar füge die Union in jedem Kooperationsvertrag mit China ihre Demokratieklausel ein; dies habe jedoch keine konsequente Demokratieförderstrategie auf Seiten der Union zur Folge. Außerdem würde China eine strikt durchgesetzte Demokratie-Konditionalität schlicht nicht akzeptieren. Algieris Fazit lautete: „Economics matters more than everything else." Solange die Mitgliedsstaaten zudem jeweils eine eigene Chinapolitik verfolgten, werde sich daran nichts ändern.

\section{Sicherheit bleibt oberste Priorität im Mittel- meerraum}

Annette Jünemann beobachtet eine inkohärente Politik der Europäischen Union gegenüber den südlichen Mittelmeeranrainern, sowohl im Rahmen der Euro-Mediterranen Partnerschaft als auch im Rahmen der Nachbarschaftspolitik. Die Union lege großen Wert auf die ökonomische Entwicklung und die Verbreitung von Demokratie im südlichen Mittelmeerraum mit dem Ziel, aktuelle Sicherheitsrisiken ursächlich zu bekämpfen. Allerdings würde die politische Konditionalisierung der europäischen Wirtschafts- und Finanzhilfe seitens der Union nicht implementiert und damit die Reformverweigerung der Regierungen im südlichen Mittelmeerraum nicht sanktioniert. Eine Chance zur Demokratieförderung bestünde in der Unterstützung zivilgesellschaftlicher Akteure, die als ,change agents' wirken könnten. Allerdings würden, so Jünemann, gerade die EU-Programme zur Förderung der Zivilgesellschaften in den letzten Jahren immer weniger auf eine direkte Förderung der Demokratisierung hin angelegt. Diese Kluft zwischen Anspruch und Wirklichkeit erklärt Jünemann mit Rollenkonflikten gemäß des konstruktivistischen Ansatzes der außenpolitischen Rollentheorie: Die außenpolitische Rolle der EU als ,Wahrer von Demokratie und Menschenrechten ' konfligiere mit ihrer außenpolitischen Rolle als ,sicherheitspolitischer Akteur' sowie mit der Rolle einzelner Mitgliedstaaten, die sich als ,Wahrer nationaler Interessen ‘ verstünden.
Richard Youngs beobachtet ein ähnliches Auseinanderklaffen zwischen Rhetorik und tatsächlichem Handeln der Europäischen Union gegenüber dem Mittleren Osten. Die Verträge und Verlautbarungen der Union seien auf Demokratieförderung ausgerichtet. Das tatsächliche Handeln der Union bekämpfe dagegen fast ausschließlich Symptome wie Sicherheitsbedrohungen durch den verstärkt wahrgenommenen Terrorismus nach 9/11. Einen wichtigen Beitrag zur Stabilisierung der Region mittels einer aktiven Demokratieförderung kann Youngs nicht erkennen, zumal die finanziellen Mittel für solche Maßnahmen nach den Anschlägen auf das World Trade Center nicht wesentlich erhöht worden seien. Die Union agiere weitgehend als, Juniorpartner" der USA.

Einen kritischen Einblick in die Menschenrechtspolitik der Europäischen Union im Nahen Osten aus der Sicht des Praktikers gewährte Marc Schade-Poulsen. Seiner Ansicht nach hat sich die Menschenrechtssituation in den letzten zehn Jahren nicht verändert. Ungeachtet vieler Reformbemühungen habe die Union beispielsweise 2005 kein einziges Programm zur Menschenrechtsförderung im Nahen Osten aufgelegt, trotz vieler Reden zum Thema und trotz des Barcelona-Prozesses. Die anschließende Debatte unter den Tagungsteilnehmern verdeutlichte, in welchem Ausmaß die Union auf die Bereitschaft der Zielländer angewiesen ist, Reformen im Menschenrechtsbereich von sich aus aktiv durchzuführen. Ohne diese Bereitschaft vor Ort könne die Europäische Union nichts ausrichten.

Im abschließenden Vortrag verglich Elena Baracani drei unterschiedliche Formen von Konditionalität und deren Wirkung auf die Verbesserung von Demokratie und Rechtsstaatlichkeit. Die Türkei unterliege bereits der ,pre-accession conditionality " und arbeite an der Umsetzung des acquis communautaire. Serbien-Montenegro nehme am Stabilisierungs- und Assoziierungsprozess der Union für den Westbalkan teil. Eine Annäherung an 
die Union solle dabei stufenweise und analog zur Erfüllung verschiedener Bedingungen im wirtschaftlichen und politischen Bereich erfolgen, wobei die Übernahme von Eigenverantwortung gemäß des Ownership-Prinzips im Mittelpunkt des Wiederaufbaus sowie der politischen, wirtschaftlichen und gesellschaftlichen Konsolidierung stehe. Marokko partizipiere an der neuen europäischen Nachbarschaftspolitik. Aktionspläne legten Formen wirtschaftlicher und diplomatischer Zusammenarbeit fest, ohne allerdings eine Beitrittsperspektive zu eröffnen. Baracani bestätigte die These, wonach die Aussicht auf Mitgliedschaft der effektivste positive Anreiz für Liberalisierung und Demokratisierung sei. In ihrem Modell spielen zur Erklärung der Wirksamkeit von Konditionalität lokale Akteure und mögliche Alternativen zu Verhandlungen mit der Union eine zentrale Rolle.

\section{Konferenzresümee}

Die Konferenz offenbarte ein breites Spektrum der europäischen Demokratieförderung und verdeutlichte den Teilnehmern die Notwendigkeit weiterer Klärung und Systematisierung. Die in der Literatur vertretene These, wonach die Union nur eine einzige Demokratieförderstrategie verfolge, konnten die Referenten nicht bestätigen. Vielmehr diagnostizierten die Vortragenden in ihren Beiträgen eine breite Vielfalt verschiedener Strategien sowie den kontextangepassten Einsatz unterschiedlicher Instrumente.

Annette Jünemann und Michèle Knodt reflektierten abschließend ihre Eingangshypothesen und sahen ihre These bestätigt, wonach die Steigerung bilateraler Beziehungen zwischen einem Drittstaat und zumindest einigen der EU-Mitgliedstaaten den Verzicht der Implementation negativer Instrumente nach sich ziehe. Allerdings zeigt das Beispiel China auch, dass die Europäische Union umso zurückhaltender negative Instrumente der Demokratieförderung anwendet, je größer das wirtschaftliche Potenzial des Drittstaates in seiner Beziehung zur EU ist und je mehr diesem Staat alternative Kooperationsmöglichkeiten offen stehen. Die EU werde in diesem Fall eher eine indirekte Vernetzungsstrategie anwenden. Im Mittelmeerraum, so Knodt und Jünemann, gelte hingegen die These: ,Je unsicherer ein Land, desto eher wird die EU positive politische Instrumente wählen und keine ,Reformimpulse von unten ' unterstützen, die etwa die Stabilität des Drittstaates gefährden könnten." Allerdings hat sich auch gezeigt, dass das Sicherheitsbedürfnis der Europäischen Union gerade in der neuen Nachbarschaftspolitik durchaus zu einer härtern Gangart in Sachen Konditionalität führen kann.

Skeptisch äußerten sich alle Regionalexperten zur Wirksamkeit der Demokratieklausel in Kooperationsverträgen. Solange eine Nichtimplementierung ohne Folgen für die Politik der Europäischen Union gegenüber den jeweiligen Partnern bleibt, wird die europäische Demokratieförderung mittels einer politischen Konditionalität wirkungslos bleiben. Obwohl die Tagung die Frage der Effektivität der Demokratieförderung nicht zentral in den Blick genommen hat, hoben alle Beiträge und Diskussionen die Beitrittskonditionalität als effektivstes Instrument der Demokratieförderung hervor.

Die Tagung eröffnete weiteren Forschungsbedarf vor allem in zweierlei Hinsicht: Zum einen in einer vertieften Erklärung des Instrumenteneinsatzes; zum anderen in einer stärkeren Wirkungsanalyse von Demokratieförderung. Die Formulierung adäquater Demokratieförderstrategien für spezifische Kontexte wird dementsprechend ein wichtiges Thema sowohl auf der politischen Agenda der Europäischen Union als auch auf der Forschungsagenda bleiben. Als Gewinn bringend hat sich auf der Tagung die enge Kooperation von Integrationswissenschaftlern und Regionalexperten erwiesen. Derartige Kooperationen sollten in Zukunft in komparativ angelegten Projekten weitergeführt werden. 